\title{
CULTURA DE SEGURANÇA E PRÁTICAS DE APRENDIZAGEM ORGANIZACIONAL EM ORGANIZAÇÃO DE ALTA CONFIABILIDADE
}

\author{
L. H. N. NOBRE ${ }^{1 *}$, E. S. SIQUEIRA ${ }^{1}$, L. H. NEPOMUCENO ${ }^{1}$ e V. MEYER JUNIOR ${ }^{2}$ \\ ${ }^{1}$ Universidade Federal Rural do Semi-Árido - UFERSA \\ ${ }^{2}$ Pontifícia Universidade Católica do Paraná - PUCPR \\ liananobre@ufersa.edu.br*
}

Artigo submetido em janeiro/2016 e aceito em outubro/2016

DOI: $10.15628 /$ holos.2016.3956

\section{RESUMO}

$\mathrm{O}$ artigo analisa elementos da cultura de segurança e práticas de aprendizagem organizacional em uma usina nuclear norte-americana. Realizou-se pesquisa qualitativa, descritiva, com uso de entrevista estruturada. A análise destacou três fatores: (1) caracterização das organizações de alta confiabilidade, (2) cultura de segurança, e (3) práticas de aprendizagem organizacional. Os resultados apontam para a caracterização da usina como organização de alta confiabilidade. Sistemas de informação, de segurança e envolvimento dos empregados foram os elementos de cultura destacados. Em relação às práticas de aprendizagem organizacional, distinguiram-se as formas de comunicação. Os resultados parecem convergir com os estudos que apontam que práticas de aprendizagem organizacional fortalecem a cultura de segurança.

PALAVRAS-CHAVE: Organizações de Alta Confiabilidade, Cultura de Segurança, Aprendizagem Organizacional, Cultura Organizacional, Práticas de Aprendizagem.

\section{SAFETY CULTURE AND PRACTICES OF ORGANIZATIONAL LEARNING IN A HIGH RELIABILITY ORGANIZATION}

\begin{abstract}
The paper analyzes elements of safety culture and organizational learning practices in a US nuclear power plant. A qualitative and descriptive research was conducted with the use of a structured interview. The analysis highlights three factors: (1) characterization of high reliability organizations, (2) safety culture, and (3) organizational learning practices. The results point to
\end{abstract}

characterize the plant as high reliability organization. Information systems security and involvement of employees were considered as important culture elements. In respect of organizational learning practices, highlights were the forms of communication. It was concluded that organizational learning practices strengthen the safety culture.

KEYWORDS: High Reliability Organizations, Security culture, Organizational learning, Organizational culture, Learning practices. 


\section{INTRODUÇÃO}

A maioria das empresas é estimulada a promover contínuas mudanças nos seus processos, estruturas, tecnologias e estratégias. Existe, porém, um conjunto de empresas, categorizadas como organizações que operam tecnologias de alto risco, que trabalha com processos de mudança mais lentos e graduais. Falhas e acidentes, neste tipo de organização, podem gerar consequências graves para o próprio processo produtivo, para a organização, para a sociedade e o meio ambiente, e o custo da falha é extremamente caro (MURO; MEYER JR, 2011; HALES; CHAKRAVORTY, 2015). Estes sistemas de alto risco se caracterizam pela natureza imprevisível, grande quantidade das interações de seus subsistemas e pela firme articulação das etapas dos seus processos (PERROW, 1999).

Os sistemas de alto risco são caracterizados pelos autores em diferentes perspectivas. Saleh et al (2010) apontam dois pilares que configuram estas organizações, são eles: a forma que estas abordam a prevenção e a questão da resiliência. Muro e Meyer Jr. (2011) destacam a inseparabilidade das partes destas organizações e a não linearidade de suas interações, para estes autores, analisar um subsistema de maneira separado dos demais não permite a real compreensão do mesmo, pois a análise fragmentada ignora os impactos que este tem sobre os demais subsistemas e vice-versa. Outra característica dos sistemas de alto risco está ligada à articulação das etapas do processo produtivo: em sistemas firmemente articulados não há grandes lacunas no processo produtivo e a sequência do processo é praticamente invariável (PERROW, 1999). Portanto, o gerenciamento de organizações dessa natureza demanda um processo de aprendizagem permanente, visto que imprevisibilidade, necessidade de articulação, não linearidade, são características constantes desses sistemas.

O gerenciamento organizações de alta confiabilidade demanda novos paradigmas de gestão e uma das correntes é a que trata da questão de sistemas de segurança em organizações que operam tecnologias de alto risco. Esta teoria surgiu da observação de que haviam organizações que operavam com tecnologias de alto risco com baixa ou nenhuma incidência de acidentes (MURO; MEYER JR, 2011). Nestes sistemas complexos e firmemente articulados, a principal característica da aprendizagem organizacional (AO) não é ser um propulsor da mudança organizacional, mas um processo de reflexão e ação voltado para a solução de problemas concretos no contexto das empresas, como sugerem Argyris e Schon (1978). Esta abordagem sobre AO é corroborada por Maguire, Allen e McKelvey (2011) que destacam a importância da comunicação e da linguagem como direcionadores da ação em sistemas complexos.

Outro aspecto a ser considerado é a gestão da cultura de segurança que tem se destacado como um importante fator na manutenção do bom desempenho das organizações de alta confiabilidade (COX; JONES; COLLINSON, 2006). Neste mesmo sentido, Bagnara, Parlangeli e Tartaglia (2010) ressaltam a importância de se mover de uma cultura na qual se enfatiza a culpabilidade em favor de uma cultura de segurança que promova o aperfeiçoamento dos sistemas e processos. Lekka (2011) pondera se as características que diferenciam as High Realiable Organizations - HROs das empresas comuns se originam nos processos de socialização e nos sistemas de comunicação que promovam a cultura de segurança. 
Assim, esse estudo tem como questão de pesquisa como os elementos da cultura de segurança e as práticas de AO se manifestam em uma usina nuclear norte-americana? Para responder a esta pergunta foi realizada uma entrevista qualitativa com um sujeito considerado informante privilegiado.

\section{REVISÃO BIBLIOGRÁFICA}

A teoria da alta confiabilidade (high reliability theory - HRT) surgiu partir da identificação de organizações que operam com tecnologias de alto risco, e que, ainda assim, tem baixos níveis de acidentes. Ela se baseia na crença de que os acidentes podem ser evitados através de um design organizacional que promove a reflexão e integra a responsabilização nos diversos níveis organizacionais (SAMUELS, 2010; LEKKA, 2011). Estas organizações ficaram conhecidas como organizações de alta confiabilidade (high realiable organizations - HRO), e são ambientes em que imperam a incerteza, mas fornecem a infraestrutura cognitiva que permite a aprendizagem adaptativa simultânea e desempenho confiável (WEICK; SUTCLIFFE; OBSTFELD, 1999; LEKKA, 2011; HALES; CHAKRAVORTY, 2015; ANDRIULO ET AL, 2015).

De maneira geral, as HRO's são sistemas complexos que operam com tecnologia de alto risco, com desempenho confiável. Segundo Roberts e Rousseau (1989), para uma organização ser classificada como HRO, ela deve apresentar determinadas características que as distinguem das demais organizações complexas: (a) hipercomplexidade: extrema variedade de componentes sociotécnicos, que se relacionam de forma simultânea e recursiva; (b) firme articulação e interdependência entre as muitas etapas do processo produtivo; (c) diferenciação hierárquica extrema com mecanismos descentralizados de controle e regulação; (d) redundância em sistemas de controles e de informação; (e) alta frequência de processos de checagem a partir das decisões tomadas; (f) alto grau de envolvimento e responsabilidade dos funcionários com o processo. De forma convergente, Boin e Schulman (2008) afirmam que a confiabilidade nas HRO's é alcançada através de uma preocupação constante com os processos organizacionais essenciais, manutenção do estado de alerta, tomada de decisões críticas, partilha de informação, capacidade de percepção, preocupação, e de ação. Embora não haja um consenso sobre o uso do termo confiabilidade, ou quais características realmente definem uma HRO, um conjunto de cinco práticas comuns são frequentemente utilizadas como categorias de análise destas organizações (NAVARRO, 2011; SUTCLIFFE, 2011). Estas práticas foram identificadas por Weick e Sutcliffe (2007) e estão descritas no Quadro 1.

Quadro 1: Características das práticas verificadas nas organizações de alta confiabilidade

\begin{tabular}{|l|l|}
\hline \multicolumn{1}{|c|}{ Práticas } & \multicolumn{1}{c|}{ Descrição } \\
\hline Preocupação com o fracasso & $\begin{array}{l}\text { Não subestimam pequenas falhas e as entendem como um sintoma de que } \\
\text { algo está errado, tentando compreender o sistema atualmente (dinâmica } \\
\text { organizacional). }\end{array}$ \\
\hline Relutância em aceitar & $\begin{array}{l}\text { HRO sabem que o mundo é complexo, instável, desconhecido e } \\
\text { imprevisível. Não subestimam a complexidade e tentam compreender os } \\
\text { simplificações } \\
\text { debate com pessoas de diferentes pontos de vista, sem destruir as } \\
\text { diversidades. } \\
\text { Mais visão com menor simplificação. }\end{array}$ \\
\hline
\end{tabular}




\begin{tabular}{|l|l|}
\hline Sensibilidade para as operações & $\begin{array}{l}\text { Importância às operações de como o trabalho é realizado - entender que } \\
\text { os eventos são situacionais (dinâmicos) e que existem 'falhas latentes' no } \\
\text { operacional. }\end{array}$ \\
\hline Compromisso com a resiliência & $\begin{array}{l}\text { Entendem que nenhum sistema é perfeito. Resiliência é uma combinação } \\
\text { de pequenos erros e soluções improvisadas que fazem o sistema funcionar. } \\
\text { Para isso é preciso o trabalho de experts, pessoas com experiência, } \\
\text { habilidade de recombinação e treinamento em pessoas com características } \\
\text { de serem mentalmente estimulantes. }\end{array}$ \\
\hline Deferência à expertise & $\begin{array}{l}\text { Migração da hierarquia/liderança para pessoas com maior expertise na } \\
\text { área, conforme a situação. Decisões são tomadas na linha de frente, por } \\
\text { pessoas com condições de resolver a situação e não necessariamente } \\
\text { obedece a hierarquia. }\end{array}$ \\
\hline
\end{tabular}

Fonte: WEICK e SUTCLIFFE, 2007

Outro importante conceito desenvolvido por Weick, Sutcliffe e Obstfeld (1999) que caracteriza as organizações de alta confiabilidade é o de mindfullness, que pode ser traduzido como atenção plena - um estado de vigilância e consciência sobre o que se passa. Este estado, para os autores, é viabilizado através de uma cultura organizacional que valoriza a ação somente mediante à compreensão completa do contexto em que a situação-problema ocorre. Percebe-se que as HRO's incorporam um compromisso organizacional com a segurança por meio de inúmeras verificações e mecanismos para monitorar e reportar os menores sinais de falha no sistema, aliados com uma orientação para a cultura de aprendizagem organizacional (SAMUELS, 2010; LEKKA, 2011; ANDRIULO et al, 2015).

Estas organizações partilham ainda outras características: tais como o amplo treinamento e o poder discricionário descentralizado para os funcionários da linha de frente, evitando o excesso de formalização que inibe a flexibilidade em situações críticas (WEICK; SUTCLIFFE, 2006). Percebese a importância da cultura no processo dinâmico de estruturação de uma organização de alta confiabilidade já que as mesmas tendem a investir - e depender - em valores e práticas culturais tais como respeito mútuo, diligência, mente coletiva, aprendizagem pela experiência, improvisação, construção de sentido (sensemaking) e manutenção de dúvida, orientações muito distantes da lógica instrumental. Lekka (2011) aponta que a orientação para a aprendizagem organizacional, nestas organizações, deve envolver (1) treinamento técnico continuado; (2) canais abertos de comunicação; (3) análise da causa principal de acidentes e incidentes; e (4) revisões de processos.

Schlienger e Teufel (2002), assim como Ruighaver, Maynard e Chang (2007), apontam que o estudo da cultura de segurança não deve ser isolado do estudo da cultura organizacional; e estes destacam aspectos como confiança, racionalidade, motivação e orientação para o trabalho como alguns dos pilares para a construção desta cultura de segurança. Esta indissociabilidade entre a cultura organizacional e a cultura de segurança é compartilhada por Pereira (2012, p. 7), que define cultura de segurança como "um conjunto de valores, percepções, atitudes e padrões de comportamento em relação à segurança compartilhada por membros da organização" e elenca seis atributos de segurança apresentados e descritos no quadro 2 que traz, também, os indicadores dos atributos apontados pelo autor. 
Quadro 2: Elementos da cultura de segurança

\begin{tabular}{|c|c|c|}
\hline Atributos de segurança & Descrição & Indicadores \\
\hline Ambiente organizacional & $\begin{array}{l}\text { Está relacionado com o grau de } \\
\text { comprometimento dos gestores com a } \\
\text { segurança dos trabalhadores }\end{array}$ & $\begin{array}{l}\text { Condições de trabalho } \\
\text { Nível de conforto } \\
\text { Envolvimento da liderança } \\
\text { Estabilidade } \\
\text { Continuidade }\end{array}$ \\
\hline $\begin{array}{l}\text { Sistema de Informação } \\
\text { de Segurança }\end{array}$ & $\begin{array}{l}\text { Está relacionado com a transferência de } \\
\text { informações sobre os riscos no ambiente } \\
\text { de trabalho e a maneira correta de } \\
\text { combatê-los }\end{array}$ & $\begin{array}{l}\text { Sistemas de coleta, análise e difusão } \\
\text { de incidentes e quase acidentes } \\
\text { Comunicação organizacional } \\
\text { Política de segurança }\end{array}$ \\
\hline $\begin{array}{l}\text { Envolvimento de } \\
\text { empregados }\end{array}$ & $\begin{array}{l}\text { Está relacionado com o grau do } \\
\text { comprometimento dos empregados com } \\
\text { os procedimentos de segurança e é uma } \\
\text { medida de participação na melhoria de } \\
\text { condições de trabalho e redução de erros } \\
\text { em sistemas complexos. }\end{array}$ & $\begin{array}{l}\text { Sistema de recompensas } \\
\text { Cultura de confiança }\end{array}$ \\
\hline Gestão do Conhecimento & $\begin{array}{l}\text { Está relacionado com a facilitação do } \\
\text { compartilhamento das lições aprendidas. }\end{array}$ & $\begin{array}{l}\text { Vontade e competência para tirar as } \\
\text { conclusões corretas a partir do } \\
\text { sistema de segurança } \\
\text { Disposição para mudança quando esta } \\
\text { for necessária } \\
\text { Compartilhamento de lições } \\
\text { aprendidas. }\end{array}$ \\
\hline $\begin{array}{l}\text { Aprendizado } \\
\text { Organizacional }\end{array}$ & $\begin{array}{l}\text { Está relacionado com a formação dos } \\
\text { funcionários }\end{array}$ & $\begin{array}{l}\text { Comunicação dentro da equipe de } \\
\text { trabalho } \\
\text { Busca de conhecimento } \\
\text { Capacitação }\end{array}$ \\
\hline Comportamento proativo & $\begin{array}{l}\text { Está relacionado com um ambiente } \\
\text { colaborativo e flexível, onde os } \\
\text { funcionários têm abertura para discutir e } \\
\text { questionar }\end{array}$ & $\begin{array}{l}\text { Comportamento de proatividade dos } \\
\text { funcionários no relato de erros e com } \\
\text { a política de segurança da empresa }\end{array}$ \\
\hline
\end{tabular}

Fonte: Adaptado de Pereira (2012)

Cox, Jones e Collinson (2006) apontam que a cultura de segurança é o produto de um número de subculturas interdependentes que se articulam de certa forma. Esta visão é partilhada por Chia et al (2003), para quem a cultura de segurança pode ser vista de diferentes pontos de vista, gerados por diferentes subculturas, mas que partilham valores e crenças sobre segurança, que fundamentam as normas e direcionam os comportamentos individuais e dos grupos nas organizações. Para fortalecer esta cultura de segurança, Bagnara, Parlangeli e Tartaglia (2010) enfatizam a combinação de três atividades - relatar (identificação e relato das condições adversas), auditar (análise e busca por soluções) e gerenciar (teste, implementação e divulgação das melhorias), já Shadden (2012) ressalta a importância de incorporar a segurança nos processos diários como forma de construção da cultura de segurança. Embora a AO seja apenas uma das categorias de análise proposta por Pereira (2012), este item tem sido apontado como um dos principais aspectos das organizações de alta confiabilidade, assim é preciso discuti-lo com atenção. 
Aprendizagem Organizacional tem sido objeto de estudo sob as mais diversas perspectivas e com grande fragmentação teórica (VERSIANI; FISCHER, 2009), envolvendo diversos campos: psicológicos, sociológicos, culturais, históricos, metodológicos e de gestão (ANTONELLO; GODOY, 2010; RUAS; ANTONELLO, 2003). Pawlosky (2001) categoriza a AO e suas definições em cinco perspectivas: cognitiva e do conhecimento, da tomada de decisão organizacional e da adaptação, da teoria dos sistemas, perspectiva cultural, e perspectiva da aprendizagem na ação. Antonello (2005) elenca os elementos conceituais comuns às diversas definições, quais sejam: (1) processo visão da aprendizagem como um fluxo contínuo e espiralar; (2) mudança - especificamente no que se refere à mudança de atitudes; (3) grupo - enfatizando a interação do indivíduo com o seu coletivo; (4) criação e reflexão - processo que permite a internalização e externalização, promovendo a inovação e conscientização; (5) ação - ressaltando a importância da experienciação, vivência e compartilhamento de experiências; (6) "situação" - que situa o processo de aprendizagem em um contexto, atividade e cultura; e (7) cultura - pela construção de significados e partilha, pelo sentido que se dá às experiências.

Independente da perspectiva que se escolha para trabalhar a $A O$, ressalta-se que esta engloba não só um conjunto de conteúdos, mas principalmente processos; e, para ser autêntica, a AO deve surgir do próprio fazer, por meio do acionamento e aplicação de conhecimentos prévios e da disseminação e partilha das melhores práticas (ANTONELLO, 2005; COSTELLA et al, 2009). Entretanto é relevante destacar que existem autores na área da administração apontando as limitações e dificuldades dos processos de AO. Telles e Teixeira (2004) ressaltam três tipos de argumentação que sustentam esta discussão: a) assume como impossível capturar, codificar e gerir conhecimento tácito alheio, posto que este está intimamente vinculado à experiência pessoal; b) considera que, ainda que o conhecimento pudesse ser sistematicamente mapeado, a codificação das experiências prévias não seria suficiente para auxiliar os processos de inovação e criação; c) aponta que a gestão sistemática do conhecimento possui uma essência totalitarista, proporcionando uma excessiva visibilidade do indivíduo. Além disso, Weick e Westley (2004) ressaltam a direção oposta que percorrem aprendizagem e organização já que organizar e aprender são, a princípio, processos antagônicos, pois um busca reduzir a variedade enquanto o outro se caracteriza justamente pela despadronização e aumento da variedade. Apesar destas discussões, é relevante para a dinâmica e a teoria organizacional não só definir $A O$, mas conhecer e sistematizar como esta ocorre. Colussi, Becker e Oliveira (2009) propuseram algumas práticas de aprendizagem, discriminadas no Quadro 3 e que, segundo os autores, são catalisadoras deste processo, e que quanto maior a incidência destas práticas no ambiente organizacional, maior probabilidade de que ocorra a aprendizagem.

Quadro 3: Práticas de aprendizagem e suas características

\begin{tabular}{|l|l|}
\hline \multicolumn{1}{|c|}{ Práticas de aprendizagem } & \multicolumn{1}{c|}{ Características } \\
\hline Reuniões para reflexão & $\begin{array}{l}\text { Momento para refletir a partir de experiências anteriores e estímulo à troca de } \\
\text { ideias. Pré-disposição para aprender com o passado. }\end{array}$ \\
\hline Estudos de caso & $\begin{array}{l}\text { Oportunidades para descrição de problemas organizacionais e questionamento } \\
\text { acerca de premissas existentes. }\end{array}$ \\
\hline $\begin{array}{l}\text { Planejamento através de } \\
\text { cenários }\end{array}$ & $\begin{array}{l}\text { Mudança ou suspensão de regras vigentes; estimular a experimentação por } \\
\text { meio de diferentes cenários e a capacidade dos seres humanos de transmitirem } \\
\text { informações. }\end{array}$ \\
\hline Práticas de benchmarking & $\begin{array}{l}\text { Oportunidade para uma empresa aprender com a experiência de outras, } \\
\text { questionando o que fazem, por que fazem de determinada forma e por que } \\
\text { devem mudar. }\end{array}$ \\
\hline
\end{tabular}




\begin{tabular}{|l|l|}
\hline Alianças estratégicas & $\begin{array}{l}\text { Troca de habilidades, tecnologias, competências essenciais e diretrizes } \\
\text { estratégicas entre empresas. Flexibilidade para mudar com o negócio e } \\
\text { permitir a cada parceiro aprender e sobreviver. }\end{array}$ \\
\hline Equipes autodirigidas & $\begin{array}{l}\text { Funcionários conduzem sua própria aprendizagem. Compartilhamento de } \\
\text { ideias, habilidades, objetivos e recompensas; uns aprendem com os outros ao } \\
\text { trabalharem em equipe. }\end{array}$ \\
\hline Gestão de recursos humanos & $\begin{array}{l}\text { Reconhecimento da importância do indivíduo e na organização, com } \\
\text { desenvolvimento de um ambiente que fomente/impulsione o seu aprendizado }\end{array}$ \\
\hline Comunidades de prática & $\begin{array}{l}\text { Aplicação prática do aprendido, tendo por base a troca de informações e o } \\
\text { compartilhamento de ideias, experiências e conhecimento }\end{array}$ \\
\hline
\end{tabular}

Fonte: Colussi, Becker e Oliveira (2009)

\section{METODOLOGIA}

Tendo em vista a consecução dos objetivos da pesquisa, realizou-se um estudo qualitativo e descritivo. Trata-se de um estudo qualitativo posto que se coloca entre as pesquisas que pretendem descrever problemas complexos, a interação de certas variáveis e o contexto do problema, além de compreender e classificar processos dinâmicos vivenciados pelos grupos e facilitar o entendimento das particularidades do comportamento dos indivíduos (BARROS; LEHFELD 1990, p. 52) estudando valores, percepções e motivações sem a pretensão de generalização (GONÇALVES; MEIRELLES, 2004). O caráter descritivo da pesquisa se apresenta na busca de caracterizar o fenômeno e a relação entre as variáveis sem inferir relações de causalidade entre os elementos discutidos (GIL, 2002; VERGARA, 2000).

A escolha de uma usina nuclear foi motivada pela característica da organização, citada como um sistema complexo de tecnologia de alto risco (SPEIER et al., 2011; SUTCLIFFE, 2011; COX; JONES; COLLINSON, 2006). O sujeito da pesquisa foi selecionado a partir do julgamento de que constituía caso típico no qual se tinha interesse: ele trabalha na indústria nuclear há 41 anos, sendo 27 anos na atual usina, tendo atuado em diversas atividades e departamentos, inclusive no planejamento de emergências e atualmente, gerencia o setor de Tecnologia da Informação da usina em questão.

A coleta de dados ocorreu por meio de entrevista estruturada, que foi enviada por e-mail para o respondente e este a devolveu em cinco dias. Dada a diferença de idiomas, foi feito uma checagem junto ao entrevistado, via Skype, para confirmar a compreensão das respostas dadas. A escolha da entrevista estruturada escrita definiu-se pela necessidade de dados rigorosos e coerentes e a validação via Skype se mostrou um recurso válido para garantir a pertinência da análise. O uso do Skype em coletas de pesquisas científicas tem se ampliado (LIRA; ARAGÃO; MERCADO, 2010; NICOLACIDA-COSTA; ROMÃO-DIAS; LUCCIO, 2009).

A partir da abordagem teórica relativa ao tema, foram definidas as categorias de análise, relacionadas aos três aspectos deste estudo: (1) à caracterização das organizações de alta confiabilidade, como proposto por Weick e Sutcliffe (2007), (2) à cultura de segurança, conforme Pereira (2012) e (3) às práticas de AO, propostas por Colussi, Becker e Oliveira (2009).

Os dados levantados foram comparados com os referenciais conceituais com o objetivo de identificar se a organização objeto do estudo se caracterizava como uma organização de alta confiabilidade para então proceder a análise principal da pesquisa, sobre a cultura de segurança e as práticas de $\mathrm{AO}$. Utilizou-se o Atlas $\mathrm{TI}^{\oplus}$ na versão 6.2 como ferramenta no processo de categorização da entrevista. 0 quadro 4, no apêndice, mostra uma seleção de assertivas (An) do entrevistado, que sustentam a análise. 


\section{RESULTADOS E DISCUSSÕES}

A princípio, foi feita uma leitura da entrevista, com o objetivo de checar a tradução e compreensão de todos os termos utilizados pelo entrevistado. Após esta averiguação, procedeuse a análise do conteúdo da entrevista. Em uma primeira análise, foram observados os termos mais utilizados pelo entrevistado. Foram retiradas as palavras de ligação, preposições e artigos. Os termos com maior frequência estão detalhados na Figura 1.

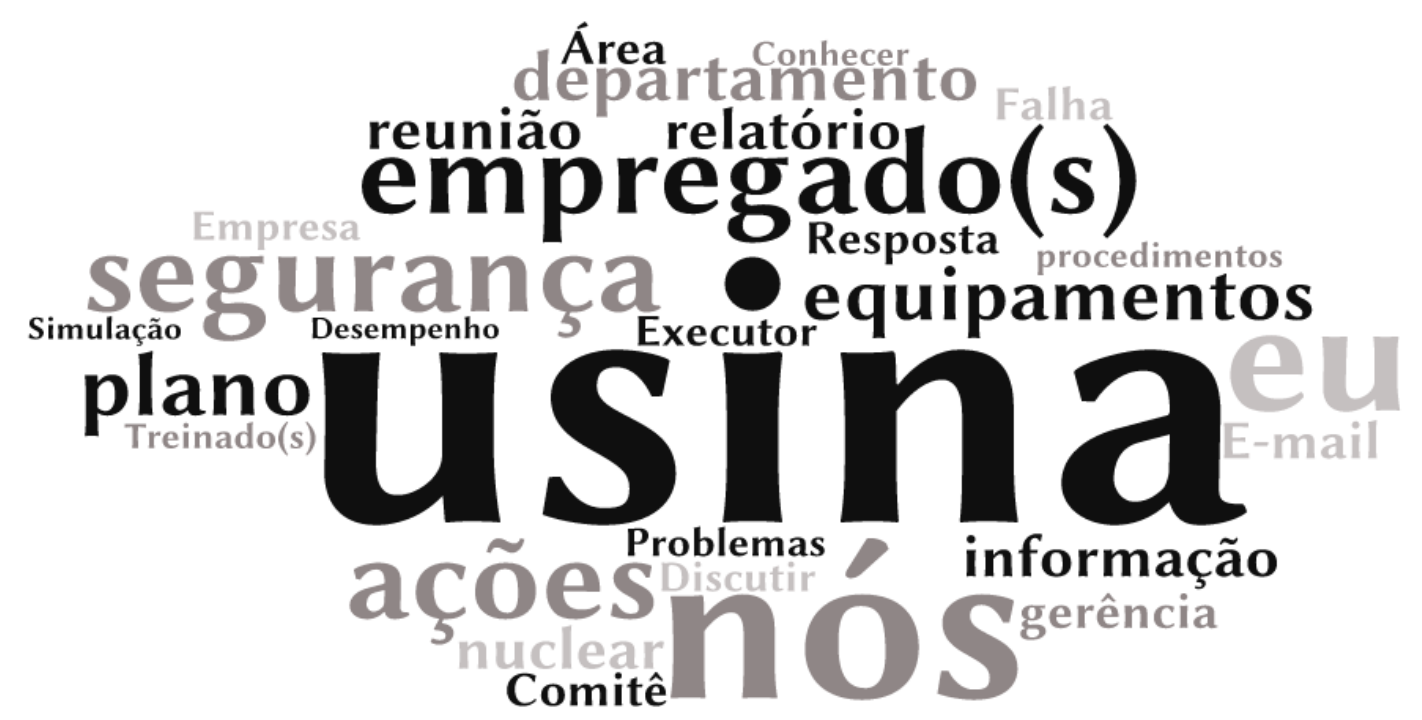

Figura 1: Nuvem de palavras com os termos mais citados na entrevista

Como se observa na Figura 1, existe uma alta incidência de referência aos "empregados", "segurança" e "informação", que são elementos importantes para a cultura de segurança. A alta frequência do termo "equipamentos" retrata que organização opera com uma tecnologia diferenciada. Embora as perguntas fossem direcionadas ao entrevistado, detectou-se também uma maior incidência de respostas no coletivo, o que sugere que o entrevistado se coloca enquanto membro de uma comunidade ou equipe. Também entre os termos mais citados, podese destacar alguns indícios da importância dos processos de comunicação e de aprendizagem como instrumentos da cultura de segurança (relatórios, reunião, e-mail, treinado(s), simulação, conhecer).

A usina nuclear é uma organização complexa, operando por meio de um conjunto de atividades que envolve tecnologias de alto risco e com firme articulação no processo produtivo. Os atributos que identificam as organizações de alta confiabilidade, segundo Weick e Sutcliffe (2007), foram identificados nas respostas do entrevistado. No que se refere à preocupação com as falhas, o entrevistado reporta um comportamento de alta atenção aos indícios de problemas nas operações, além da habilidade em descrever as falhas e formas de solucionar os problemas para uma posterior divulgação na organização (A1, $A 2)$. A relutância em aceitar simplificações é evidenciada pela procura por detalhes que possam descrever melhor as situações adversas (A3). Estas atividades são passíveis de ocorrência em uma cultura que encoraja estes relatos sem focar no desempenho individual, eximindo operadores da culpa em relação à falha e ressaltando os aspectos sistêmicos do processo (LEKKA, 2011).

Quanto à sensibilidade para as operações, os dados apontam o alto grau de conhecimento das operações por parte da equipe envolvida (A4). Foram evidenciados aspectos que definem o 
compromisso com a resiliência, tanto no que tange à manutenção do erro mínimo, com a descrição de uma metodologia que inibe que o executor pule etapas de um procedimento (A6), quanto à proatividade em situações de crise, que é uma afirmativa direta na qual o entrevistado afirma que os funcionários estão prontos para agir em todas as situações (A7).

Por fim, quanto deferência à expertise, o entrevistado descreveu um tipo de procedimento no qual se requer a presença de outro funcionário, de maior conhecimento, para avalizar a tarefa a ser realizada (A8). Esta prática está alinhada aos resultados de Yoo e Lee (2015), que em sua pesquisa encontraram escores mais altos de atenção (awareness) em funcionários com mais de nove anos de experiência em plantas nucleares. Tal como proposto por Samuels (2010), identificou-se o comprometimento da organização com a segurança por meio de inúmeras verificações dos sistemas (A4). O estado de atenção plena (mindfulness) destacado por Weick, Sutcliffe e Obstfeld (1999) e manutenção da dúvida (WEICK; SUTCLIFFE, 2006) são enfatizados pelo entrevistado, ao descrever a postura dos empregados no desempenho de suas funções (A10). Não foram encontrados indícios de improvisação ou formação de significados (sensemaking).

O entrevistado ressalta que o aspecto da segurança está inerente em todas as atividades da usina nuclear. Esta cultura de segurança foi identificada através dos indicadores sugeridos por Pereira (2012): ambiente organizacional, sistema de informação de segurança, envolvimento de empregados, gestão do conhecimento, aprendizado organizacional e comportamento proativo. Quanto ao ambiente organizacional, foram identificados indícios do envolvimento da gestão em atividades que reforçam a cultura de segurança, tanto em aspectos operacionais - com o relato de como a gerência se envolve nas questões operacionais em caso de falhas e manutenção (A5, $A 11)$, quanto em participação de encontros onde se discute as condições da usina (A12). $O$ fato de o entrevistado estar na empresa há 27 anos sugere a política de estabilidade na usina. Foram identificados vários trechos que mencionam os sistemas de coleta, análise e divulgação de incidentes e quase acidentes ( $A 2, A 3, A 13)$. A comunicação organizacional sobre as políticas de segurança (A16) ocorre através de manuais, boletins, e-mail, vídeos e página da intranet $(A 9, A 12$, A13, A14, A15, A16, A17, A18, A19).

Quanto ao envolvimento de empregados, o entrevistado cita o incentivo que se dá aos funcionários para que estes participem ativamente reforçando a cultura de segurança, com base no desempenho do funcionário e no baixo índice de acidentes de trabalho (A26, A28). Os funcionários são capacitados (A23) e as respostas do entrevistado destacam o grau de confiança que há entre os funcionários (A21, A22). A gestão do conhecimento pode ser identificada através do compartilhamento das lições aprendidas. Em toda a indústria nuclear, procedimentos têm sido alterados com base em aprendizados com eventos anteriores (A31). Especificamente na usina em análise, foram identificadas práticas de compartilhamento de experiências e conhecimento (A19, A31). Em relação ao comportamento proativo, foram relatados casos do próprio entrevistado, nos quais ele busca soluções para diversas questões de segurança, e partilha as mesmas com os demais membros de sua equipe (A30, $A 32)$.

A AO, enquanto categoria da cultura de segurança caracterizada por Pereira (2012), pode ser evidenciado através das reuniões diárias (A18, $A 19)$, semanais (A30, $A 32)$, trimestrais (A12), e eventuais (A3); e procedimentos de passagem de serviço (A33). Analisando as respostas do entrevistado, são identificadas diversas práticas de $A O$ além das frequentes reuniões entre os membros da mesma equipe e entre departamentos e níveis diferentes. Os estudos de caso, embora menos citados pelo entrevistado, envolvem questões ligadas à segurança tanto em usinas (A30), como em outros sistemas complexos que operam com tecnologias de risco (A32) e promovem a discussão entre os membros da equipe. 
A formação de alianças estratégicas e as práticas de benchmarking são práticas de que não podem ser plenamente analisadas em sua forma original devido a existência de um órgão regulador que estabelece normas, coleta e distribui as informações sobre segurança entre as usinas nucleares (A31). Mesmo assim, observa-se uma preocupação em analisar as experiências passadas em outras usinas como forma de aprendizagem (A29). Foram relatados aspectos ligados à gestão de pessoas, tal como o estímulo à uma atitude questionadora e à prática de reportar os incidentes (A20, A32), treinamento de procedimentos de segurança (A23, A31, A34, A35). A única iniciativa que indicam a existência de um aprendizado dirigido pela própria equipe é a relatada na assertiva 30, quando o entrevistado relata que, semanalmente, busca informações e as partilha com os membros da equipe, sendo um indicador de uma equipe autodirigida. Por fim, não foram encontrados indícios da existência de comunidades de prática ou planejamento por meio de cenários. As respostas apresentadas pelo entrevistado são, de maneira geral, impregnadas da cultura de segurança da usina. Esta cultura da preocupação com as falhas se reflete até na utilização de métodos de segurança em atividades que não envolvem alto risco, tal como relatado pelo entrevistado: "quando fazemos mudanças de software, nós usamos um método de segurança semelhante (ao método de círculos e traços)".

A informação institucional parece ser um forte elemento na cultura de segurança da usina. As diversas formas pelas quais as informações sobre segurança transitam indicam que a organização usa da redundância (PERROW, 1999) como estratégia para garantir que os membros recebam a informação. Considerando-se a estrutura de análise de culturas e subculturas proposta por Reason (1997, 1998, 2000) e sistematizada por Cox, Jones e Collinson (2006), a importância das práticas de aprendizagem para reforçar esta cultura de segurança, e esta cultura de aprendizagem está firmemente suportada por uma cultura de informação. $O$ envolvimento dos empregados também se destacou entre os elementos da cultura, principalmente no que se refere às relações de confiança entre os membros da equipe, o que é coerente com os pressupostos de Cox, Jones e Collinson (2006). Entre as práticas de aprendizagem, destacam-se as reuniões, que proporcionam a comunicação direta e favorecem a troca de experiências e a discussão dos procedimentos da organização.

\section{CONSIDERAÇÕES FINAIS}

O objetivo do estudo foi analisar os elementos da cultura de segurança e as práticas de AO em uma usina nuclear norte-americana. Apesar da limitação da pesquisa, posto que foi realizada apenas uma entrevista, apresentando assim um resultado restrito, os resultados obtidos corroboram os pressupostos teóricos ao encontrar evidências de que a cultura de segurança e a AO desempenham importante papel para a manutenção dos baixos (ou nulos) níveis de acidentes, incidentes ou falhas nas organizações de alta confiabilidade. A análise realizada parece indicar que a cultura de segurança na organização é forte e se revela nas narrativas do entrevistado, que descreve quase todos os indicadores da cultura de segurança presentes na literatura. Percebe-se, no discurso do entrevistado, uma indicação de que a subcultura da informação ampara a subcultura de aprendizagem, sendo, portanto, uma das principais bases para a sustentação e contínuo fortalecimento da cultura de segurança da empresa. Em conformidade com a linha de pesquisa utilizada neste trabalho, para os próximos estudos relacionados aos elementos de cultura de segurança e práticas de $\mathrm{AO}$, sugere-se que se faça um survey para analisar, através de análise multivariada de dados, as relações entre as categorias da pesquisa, encontrando como as subculturas de informação, aprendizagem e segurança se reforçam nas organizações de alta confiabilidade. 


\section{REFERÊNCIAS BIBLIOGRÁFICAS}

1. AMBROZ, M. Innovative Issues and Approaches in Social Sciences. Innovative Issues and Approaches in Social Sciences, v. 5, n. 1, p. 70-87, 2015.

2. ANDRIULO, S. et al. Effectiveness of maintenance approaches for high reliability organizations. IFAC Proceedings Volumes, v. 48, n. 3, p. 466-471, 2015.

3. ANTONELLO, C. S. A metamorfose da aprendizagem organizacional: uma revisão crítica. In: RUAS, R. L.; ANTONELLO, C. S.; BOFF, L. H. (org.) Os novos horizontes da gestão: aprendizagem organizacional e competências. Porto Alegre: Editora Bookman, 2005.

4. ANTONELLO, C. S.; GODOY, A. S. A Encruzilhada da Aprendizagem Organizacional: uma Visão Multiparadigmática. Revista de Administração Contemporânea, v. 14, n. 2, 2010.

5. ARGYRIS, C.; SCHÖN, D. Organizational Learning: a Theory of Action Perspective. Reading/Mass: Addison-Wesley, 1978.

6. BAGNARA, S.; PARLANGELI, O.; TARTAGLIA, R. Are hospitals becoming high reliability organizations? Applied ergonomics, v. 41, n. 5, p. 713-8, set. 2010.

7. BARROS, A. J. P.; LEHFELD, N.A.S. Projeto de Pesquisa: Propostas Metodológicas. Petrópolis: Vozes, 1990.

8. BOIN, A.; SCHULMAN, P. Assessing NASA's Safety Culture: The Limits and Possibilities of HighReliability Theory. Public Administration Review, v. 68, n. 6, p. 1050-1062, 2008.

9. COLUSSI, D.; BECKER, G. V.; OLIVEIRA, M. A relação entre as práticas de aprendizagem organizacional e o processo de formação de estratégias em uma instituição de ensino preparatório para concurso. Estudos do CEPE, n. 29, 2009.

10. COSTELLA, M. F.; SAURIN, T. A.; GUIMARÃES, L. B. A method for assessing health and safety management systems from the resilience engineering perspective. Safety Science, 47, 10561067, 2009.

11. COX, S.; JONES, B.; COLLINSON, D. Trust relations in high-reliability organizations. Risk analysis: an official publication of the Society for Risk Analysis, v. 26, n. 5, p. 1123-38, out. 2006.

12. GIL, A. C., Como elaborar projetos de pesquisa, São Paulo, 4 ed., São Paulo: Editora Atlas, 2002.

13. GONÇALVES, C. A.; MEIRELLES, A. M. Projetos e relatórios de pesquisa em administração. São Paulo: Atlas, 2004.

14. HALES, D. N.; CHAKRAVORTY, Satya S. Creating high reliability organizations using mindfulness. Journal of Business Research, v. 69, n. 8, p. 2873-2881, 2016.

15. LEKKA, C. High reliability organizations: A review of the literature. Health and Safety Laboratory. London, 2011.

16. LIRA, M.T.V.; ARAGÃO, M.H.M.; MERCADO, L.P.L. Desafios do Skype na Coleta de Dados na Pesquisa em Educação Online in: V EPEAL - Encontro de Pesquisa em Educação de Alagoas, 2010, Maceió. Anais...Maceió, Universidade Federal de Alagoas.

17. MAGUIRE, S.; ALLEN, P.; MCKELVEY, B. Complexity and Management: Introducing SAGE Handbook. In: ALLEN, P.; MAGUIRE, S.; MCKELVEY, B. The SAGE Handbook of Complexity and Management. Los Angeles: SAGE, 2011. 
18. MURO, P.; MEYER JR, V. ORGANIZAÇÕES COMPLEXAS E CONFIABILIDADE ORGANIZACIIONAL: REFLEXÕES SOBRE AS HRO-HIGH RELIABILITY ORGANIZATIONS. Revista de Negócios, v. 16, n. 2, p. 86-98, 2011.

19. NAVARRO, L. L. L. Organizações de alta confiabilidade: um estudo sobre suas características e princípios. 2011. 226 p. Dissertação (Mestrado em Engenharia de Produção) - Programa de Engenharia de Produção, UFRJ/COPPE, 2011.

20. NICOLACI-DA-COSTA, Ana Maria; ROMÃO-DIAS, Daniela; DI LUCCIO, Flávia. Uso de entrevistas on-line no método de explicitação do discurso subjacente (MEDS). Psicologia: Reflexão e Crítica, v. 22, n. 1, p. 36-43, 2009.

21. PAWLOWSKY, P. Management science and organizational learning. In M. Dierkes, A. Berthoin Antal, J. Child, \& I. Nonaka (Orgs.), The handbook of organizational learning and knowledge (pp. 61-88). Oxford: Oxford University Press, 2001.

22. PERROW, C. Normal Accidents: Living with High - Risk Technologies. Princeton, NJ: Princeton University Press, 1999.

23. PEREIRA, J. Indicadores de cultura de segurança na indústria de manutenção aeronáutica. In: Simpósio de Administração da Produção, Logística e Operações Internacionais, XV, 2012, São Paulo. Anais... São Paulo: FGVEAESP, 2012.

24. ROBERTS, K. H.; ROUSSEAU, D. M. Research in nearly failure-free, high-reliability organizations: having the bubble. IEEE Transactions on Engineering Management, v. 36, n. 2, 1989.

25. RUAS, R.; ANTONELLO, C. S. Repensando os referenciais analíticos em aprendizagem organizacional: uma alternativa para análise multidimensional. Revista de Administração Contemporânea, v. 7, n. 3, p. 203-212, 2003.

26. RUIGHAVER, A. B.; MAYNARD, S. B.; CHANG, S. Organisational security culture: Extending the end-user perspective. Computers and Security, v. 26, n. 1, p. 56-62, 2007.

27. SALEH, J. H.; MARAIS, K.B.; BAKOLAS, E.; COWLAGI, R.V. Highlights from the literature on accident causation and system safety: Review of major ideas, recent contributions, and challenges. Reliability Engineering and System Safety, v. 95, p. 1105-1116, 2010.

28. SAMUELS, J. G. The application of high-reliability theory to promote pain management. The Journal of nursing administration, v. 40, n. 11, p. 471-6, 2010.

29. SCHLIENGER T.; TEUFEL, S. Information security culture - the socio- cultural dimension in information security management. In: IFIP TC11 international conference on information security, Cairo. Proceedings... Cairo, Egypt, 2002.

30. SHADDEN, M. Developing a Security Culture. Journal: American Water Works Association, v. 104, n. 6, p. 28-30, 2012.

31. SPEIER, C. et al. Global supply chain design considerations: Mitigating product safety and security risks. Journal of Operations Management, v. 29, n. 7-8, p. 721-736, 2011.

32. SUTCLIFFE, K. M. High reliability organizations (HROs). Best practice \& research. Clinical anaesthesiology, v. 25, n. 2, p. 133-44, 2011.

33. TELLES, M.M.M.; TEIXEIRA, F.L.C. Aspectos de dominação e emancipação na gestão do conhecimento organizacional: o papel da tecnologia da informação. In: Cidade de Conhecimento [online]. Disponível em: <http://www.cidade.usp.br> Acesso em: 02 de 
setembro de 2004.

34. VERGARA, S. C. Projetos e relatórios de pesquisa em administração. 3. ed, São Paulo: Atlas, 2000

35. VERSIANI, A. F.; FISCHER, A. L. A aprendizagem organizacional como um campo específico de conhecimento no cenário dos estudos organizacionais. Revista Economia \& Gestão, v. 8, n. 18, p. 10-31, 2009.

36. WEICK, K. E.; SUTCLIFFE, K. M. Mindfulness and the Quality of Organizational Attention. Organization Science, v. 17, n. 4, p. 514-524, 2006.

37. _. Managing the unexpected: resilient performance in an age of uncertainty. San Francisco: John Wiley \&Sons, 2007.

38. WEICK, K. E.; SUTCLIFFE, K.M.; OBSTFELD, D. Organizing for High Reliability: Processes of Collective Mindfulness. In: SUTTON, R.S. and STAW, B.M. Research in Organizational Behavior, Volume 1; Stanford: Jai Press, pp. 81-123, 1999.

39. WEICK, K.E.; WESTLEY, F. Aprendizagem Organizacional: confirmando um oximoro. In: CLEGG, S.R., HARDY, C., NORD, W. (org.), Handbook de estudos organizacionais. Volume 3. São Paulo: Atlas, 2004, p. 361-388.

40. YOO, H.; LEE, J. H. Results of nuclear security culture survey on personnel at nuclear power plants. Annals of Nuclear Energy, v. 85, p. 398-402, 2015.

\section{APÊNDICE}

\begin{tabular}{|c|c|}
\hline \multicolumn{2}{|r|}{ Quadro de Assertivas } \\
\hline A1 & $\begin{array}{l}\text { "Porque nós temos uma atitude de questionamento, estamos sempre à procura de condições fora do } \\
\text { normal, como indícios de água no chão onde ele deveria estar seco; ou um ligeiro cheiro de fumaça onde } \\
\text { não deveria haver nenhum cheiro; ou uma luz piscando que deveria estar acesa; ou um rangido onde } \\
\text { deveria haver o som de uma máquina bem lubrificada, ou ainda um cano quente que deveria estar em } \\
\text { temperatura ambiente." }\end{array}$ \\
\hline$A 2$ & $\begin{array}{l}\text { "Nós escrevemos relatórios de investigação que descreve as principais causas das falhas e incidentes e } \\
\text { descrevemos os métodos para garantir que isso não vai acontecer novamente." }\end{array}$ \\
\hline A3 & $\begin{array}{l}\text { "Quando equipamentos apontam o surgimento de tendências adversas que podem resultar em falhas, a } \\
\text { gerência convoca uma reunião para uma iniciativa de tomada de decisão operacional e faz planos para o } \\
\text { que fazer. Estes planos são comunicados aos funcionários da planta por e-mail. (...) quando ocorrem } \\
\text { problemas significativos com equipamentos, o e-mail enviado aos funcionários é uma página cheia de } \\
\text { informações, em vez de um parágrafo curto." }\end{array}$ \\
\hline A4 & $\begin{array}{l}\text { "Os funcionários são treinados para operar a usina em segurança. Há instrumentação em toda a planta que } \\
\text { exibe dados de temperatura, pressão, vazão, níveis de tanques, tensão, correntes e concentração química, e } \\
\text { sabemos que esses números deveriam ser. Quando certos números específicos estão fora dos limites } \\
\text { estabelecidos, os alarmes soarão e os funcionários vão agir. Os monitores exibem gráficos que mostram as } \\
\text { leituras dos instrumentos ao longo do tempo, para que os funcionários possam identificar tendências } \\
\text { adversas." }\end{array}$ \\
\hline A5 & $\begin{array}{l}\text { "A gestão da fábrica monitora o status dos indicadores de desempenho importantes ao longo de meses e } \\
\text { anos para identificar outras tendências adversas. Usamos manutenção preditiva para prever o potencial de } \\
\text { falha dos equipamentos ao longo do tempo." }\end{array}$ \\
\hline
\end{tabular}




\begin{tabular}{|c|c|}
\hline A6 & $\begin{array}{l}\text { "Certos procedimentos são classificados como procedimentos de "uso contínuo". Isso significa que o } \\
\text { executor deve ter uma cópia do procedimento à vista e abrir na página da descrição do procedimento, ou } \\
\text { estar em contato com um colega de trabalho que tem uma cópia. O executor vai ler e entender cada passo } \\
\text { antes de executá-lo, e vai executar cada etapa, como escrito na sequência. O executor vai usar um método } \\
\text { para evitar pular ou repetir um passo: ele circula a etapa depois de ler e entender, então executa a ação. } \\
\text { Em seguida, corta o círculo com um traço para indicar que a etapa foi concluída. Depois que a tarefa foi } \\
\text { concluída, ele revisa as etapas do procedimento." }\end{array}$ \\
\hline A7 & $\begin{array}{l}\text { "Eles (os empregados) reconhecem as condições que necessitam de reações específicas, e eles sabem os } \\
\text { procedimentos sobre como reagir em cada situação." }\end{array}$ \\
\hline A8 & $\begin{array}{l}\text { "Quando as ações que poderia resultar em consequências significativas são tomadas, exige-se que o } \\
\text { executor envolva um colega de trabalho mais experiente para realizar um checagem entre pares." }\end{array}$ \\
\hline A9 & $\begin{array}{l}\text { "Para cada funcionário é dado um pequeno livro de bolso intitulado "Normas e expectativas." O livro } \\
\text { contém informações que irão orientar os funcionários para um melhor desempenho nas suas atividades. Ele } \\
\text { fornece descrições de métodos que são usados para reforçar a segurança na e define os hábitos de trabalho } \\
\text { dos empregados que minimizar os erros e evitar eventos". }\end{array}$ \\
\hline A10 & $\begin{array}{l}\text { "Nós desafiamos hipóteses, investigamos anomalias e consideramos as possíveis consequências adversas de } \\
\text { ações planejadas. Nós entendemos que os acidentes podem acontecer como resultado de decisões erradas } \\
\text { devido a falhas nos pressupostos comuns da organização. Nós estamos atentos às condições e atividades } \\
\text { que possam ter um efeito indesejável sobre a segurança das instalações." }\end{array}$ \\
\hline A11 & $\begin{array}{l}\text { "A primeira coisa que acontece depois de uma falha de equipamento importante é que o equipamento é } \\
\text { colocado em "quarentena" para preservá-lo na condição em que estava quando ele falhou. Em seguida, } \\
\text { funcionários e administração criam um plano de resolução de problemas para desmontar o equipamento e } \\
\text { determinar o motivo da falha. Uma vez que a razão para a falha tiver sido detectado, a gestão vai averiguar } \\
\text { se o mesmo tipo de falha é possível em outro equipamento semelhante." }\end{array}$ \\
\hline A12 & $\begin{array}{l}\text { "A alta direção realiza uma reunião geral uma vez a cada três meses, onde todos os funcionários são } \\
\text { convidados a participar. Nós discutimos as recentes ações que tomamos, comemoramos as vitórias, e } \\
\text { falamos sobre os próximos desafios que vamos enfrentar. A reunião é gravada em vídeo e disponibilizada na } \\
\text { intranet da fábrica para os funcionários que não puderam comparecer." }\end{array}$ \\
\hline A13 & $\begin{array}{l}\text { "Se houver um acidente de ferimento na planta, mesmo os da magnitude de uma picada de abelha, este é } \\
\text { registrado no sistema de rastreamento do evento e um e-mail é enviado para os funcionários. Se uma lesão } \\
\text { requer tratamento médico por um médico externo, é chamado de uma lesão "documentável"." }\end{array}$ \\
\hline A14 & $\begin{array}{l}\text { "Boletins especiais são enviados por e-mail toda a planta se houver uma situação relacionada com a } \\
\text { segurança em andamento, por exemplo, o tráfego de pé em uma determinada área a ser restrita devido a } \\
\text { trabalhos de construção." }\end{array}$ \\
\hline A15 & $\begin{array}{l}\text { "O manual de segurança do site está disponível no site da fábrica, com acesso para todos os funcionários. O } \\
\text { site também tem "Fichas de Segurança" que descrevem os perigos e as propriedades de cada produto } \\
\text { químico que é usado no local, para que os funcionários possam conhecer os perigos que eles enfrentam } \\
\text { quando trabalham com produtos químicos." }\end{array}$ \\
\hline A16 & $\begin{array}{l}\text { "Um dos princípios que usamos é parar, pensar, agir, revisar. Antes de executar uma ação, nós paramos e } \\
\text { concentramos a nossa atenção na tarefa. Nós pensamos sobre o que será o resultado quando realizamos a } \\
\text { ação, e podemos garantir que estamos tomando a ação do componente correto. Agimos. E nós revisamos a } \\
\text { ação para nos certificarmos de que os resultados são o que esperávamos." }\end{array}$ \\
\hline A17 & $\begin{array}{l}\text { "Na Intranet da fábrica, e em grandes monitores nos principais edifícios, há uma apresentação que } \\
\text { demonstra quaisquer mudanças que possam estar acontecendo naquele dia." }\end{array}$ \\
\hline A18 & $\begin{array}{l}\text { "Cada departamento tem um rápido encontro próximo do início de cada jornada de trabalho onde } \\
\text { discutimos sobre uma mensagem de segurança, cobrindo uma ampla variedade de tópicos: nuclear, } \\
\text { radiação, e segurança industrial." }\end{array}$ \\
\hline
\end{tabular}




\begin{tabular}{|c|c|}
\hline A19 & $\begin{array}{l}\text { oda terça-feira, nós lemos e discutimos um boletim que traz informações do biênio sobre segurança e } \\
\text { sempenho humano. Cada página tem várias perguntas no final que estimulam uma discussão mais } \\
\text { rofundada sobre o assunto." }\end{array}$ \\
\hline A20 & $\begin{array}{l}\text { "Como somos encorajados a manter um saudável senso de inquietude, em vez de complacência, estamos } \\
\text { preparados para os problemas o tempo todo." }\end{array}$ \\
\hline A21 & $\begin{array}{l}\text { "Através da experiência, sabemos como a usina é como quando ela está funcionando bem. (...) Os } \\
\text { funcionários da minha empresa são totalmente capazes de perceber problemas." }\end{array}$ \\
\hline A22 & "Tenho plena confiança na reação dos funcionários da minha planta para qualquer problema." \\
\hline A23 & $\begin{array}{l}\text { "Os funcionários são treinados para operar a usina em segurança. Nosso principal dever como } \\
\text { seguros e responsáveis é proteger a saúde e a segurança do público em geral que vivem pertc }\end{array}$ \\
\hline A24 & $\begin{array}{l}\text { locar a planta em condições de segurança, } \\
\text { :a." }\end{array}$ \\
\hline A25 & $\begin{array}{l}\text { "A fábrica criou um Comitê Local de Segurança, composta por funcionários da maioria dos principais } \\
\text { departamentos. Cada funcionário permanece na comissão por pelo menos } 8 \text { meses. Empregados em cada } \\
\text { departamento conhecem seu representante, e eles são incentivados a ir até ele e fazer sugestões que ele irá } \\
\text { levar para a reunião mensal do comitê. O comitê tem um "padrinho", que é um membro da administração. } \\
\text { Isso garante que as sugestões levantadas pela comissão receberão atenção suficiente e poderão ser } \\
\text { implementadas. A comissão também tem um orçamento que eles usam para implementar as sugestões de } \\
\text { segurança, e as ações que eles implementam são rastreadas em uma lista que é disponibilizada para todos } \\
\text { os funcionários." }\end{array}$ \\
\hline A26 & $\begin{array}{l}\text { "No final do ano, o funcionário e seu gerente discutem as realizações do funcionário e como elas se } \\
\text { relacionam com o seu plano de desempenho. O funcionário tem acesso a uma página web onde o histórico } \\
\text { de seu desempenho pessoal é armazenado, e ele pode adicionar suas informações e realizações a qualquer } \\
\text { momento durante o ano. O gerente tenta classificar o desempenho do empregado de forma consistente e } \\
\text { justa. A discussão envolve métodos para melhorar o desempenho futuro e esta classificação resulta em um } \\
\text { ajuste no pagamento do funcionário, bem como em bônus anuais." }\end{array}$ \\
\hline A27 & $\begin{array}{l}\text { "A intranet da planta tem um formulário on-line disponível que qualquer funcionário pode usar para } \\
\text { escrever um relatório. O relatório pode ser sobre qualquer coisa que precisa ser analisada, e pode ser } \\
\text { preenchido de forma anônima. Estes relatórios são avaliados pela gestão da fábrica, e elencados em ordem } \\
\text { de prioridade." }\end{array}$ \\
\hline A28 & $\begin{array}{l}\text { ano, e se o número supera a } \\
\text { do." }\end{array}$ \\
\hline A29 & $\begin{array}{l}\text { "O relatório de status planta diariamente contém uma página que relata uma experiência operacional de } \\
\text { outra planta, uma espécie de "este dia na história". O boletim semanal da planta contém de um a três } \\
\text { reportagens sobre os eventos em outras plantas dos Estados Unidos, e pelo menos um artigo relatando uma } \\
\text { experiência operacional que aconteceu no passado em nossa fábrica." }\end{array}$ \\
\hline A3 & $\begin{array}{l}\text { ra, eu analiso relatos de experiência operacional nos Estados Unidos e seleciono um evento } \\
\text { compartilhar com meu departamento em nossa reunião da manhã." }\end{array}$ \\
\hline A3. & $\begin{array}{l}\text { "Os empregados da usina estão cientes e treinados sobre situações no passado onde a resposta a } \\
\text { determinadas situações em outras plantas não foi eficaz, e toda a indústria nuclear tem feito mudanças } \\
\text { para eliminar essas respostas ineficazes." }\end{array}$ \\
\hline $\mathrm{A} 3$ & $\begin{array}{l}\text { "Somos encorajados a compartilhar histórias pessoais de problemas de segurança não relacionados a usinas } \\
\text { nucleares, que encontramos por conta própria. Às vezes falamos sobre segurança náutica ou aeronaves, e } \\
\text { uma ou duas questões não são respondidas, porque nenhum de nós teve a experiência de conhecer os } \\
\text { aspectos de segurança em questão. Se há uma pergunta que paira no ar, no final da reunião, eu sempre } \\
\text { volto para minha mesa e tento encontrar a resposta, para, em seguida, enviá-la para os outros membros do } \\
\text { departamento. Às vezes, dois ou mais de nós pesquisamos a mesma pergunta, e a discussão continua } \\
\text { através de e-mails." }\end{array}$ \\
\hline
\end{tabular}




\begin{tabular}{|l|l|}
\hline A33 & $\begin{array}{l}\text { "Todos os dias, na reunião da manhã, fazemos uma revisão do estado da planta, observamos qualquer } \\
\text { equipamento importante que está em manutenção, e falamos sobre qualquer trabalho significativo que } \\
\text { está em andamento." }\end{array}$ \\
\hline A34 & $\begin{array}{l}\text { "Treinamentos de incêndio são realizados em edifícios de escritórios, pelo menos uma vez por ano. O } \\
\text { exercício envolve a ativação do alarme de incêndio e a evacuação de todos os funcionários do prédio. Os } \\
\text { treinamentos de incêndio são realizados na fábrica pelo menos uma vez por trimestre. Trata-se de uma } \\
\text { atuação do Corpo de Bombeiros na planta, com todos os equipamentos utilizados em uma situação real." }\end{array}$ \\
\hline A35 & $\begin{array}{l}\text { "O Plano de Emergência da planta é testado uma vez a cada dois anos. Estes "exercícios de emergência" são } \\
\text { observados pela Comissão Reguladora Nuclear e envolvem equipes de pessoal de emergência, as equipes } \\
\text { de reparo vão para a fábrica para simular a reparação de equipamentos danificados, simula-se a liberação } \\
\text { de radiação, o monitoramento da radiação pelas equipes de campo, a evacuação dos residentes para áreas } \\
\text { próximas, a montagem e ativação dos equipamentos de emergência e equipes de reação. Instalações de } \\
\text { emergência da planta são ativados com mais frequência para testar os tempos de resposta dos membros da } \\
\text { equipe e sua capacidade de configurar e operar sua área de especialização e utilizar os seus procedimentos. } \\
\text { Os alarmes, que tem um alcance aproximado de } 16 \text { quilômetros em torno da planta, são ativados como } \\
\text { teste pelo menos uma vez por ano." }\end{array}$ \\
\hline
\end{tabular}

